\title{
Hospital Utilization and the Respiratory Disease Season
}

\author{
Ronald Lagoe*, Shelly Littau \\ Hospital Executive Council, Syracuse, NY, USA \\ Email: ^Hospexcl@cnymail.com
}

How to cite this paper: Lagoe, R. and Littau, S. (2020) Hospital Utilization and the Respiratory Disease Season. Case Reports in Clinical Medicine, 9, 40-46. https://doi.org/10.4236/crcm.2020.91007

Received: December 6, 2019

Accepted: January 11, 2020

Published: January 14, 2020

Copyright $\odot 2020$ by author(s) and Scientific Research Publishing Inc. This work is licensed under the Creative Commons Attribution International License (CC BY 4.0).

http://creativecommons.org/licenses/by/4.0/

\begin{abstract}
In the United States, the respiratory disease season has challenged providers to care for populations at the community level. This study reviewed the utilization of inpatient hospitals in the metropolitan area of Syracuse, New York during the most recent twelve-month periods. It focused on the onset and duration of respiratory disease utilization. The study evaluated this utilization by identifying numbers of hospital patients admitted with respiratory diseases to the combined Syracuse hospitals by month. By focusing on twelve-month periods for July-June of each year, it was possible to identify changes in hospital utilization for respiratory disease season. The study demonstrated that, during the five-year period, inpatient respiratory diseases were concentrated between January and March. The highest levels of respiratory disease occurred during January. The study also demonstrated that the onset of the respiratory disease season was rapid, demonstrated by increases in census levels and discharges between October-December and January-March. The study suggested that the highest demands for inpatient care in Syracuse increased by a community wide average of 15 to 20 patients during January. The data show that increased levels of respiratory diseases extended from January-March into April and May. This approach to the utilization of health care could be employed by other providers to address the onset and duration of respiratory disease at the community level.
\end{abstract}

\section{Keywords}

Respiratory Disease, Influenza Season, Hospitals, Hospital Utilization

\section{Introduction}

The utilization of health care is closely related to the populations that it serves. This includes the demographics of these populations, as well as the incidence of diseases within them [1]. 
Demographic factors such as population age, gender, and residence tend to be rather consistent over time. The incidence of diseases can change more rapidly as a result of seasonal or local factors [2] [3].

Historically, the management of health care use has been challenged by the variability of this utilization. The allocation and the use of resources to address the needs of this population require health care managers to plan, implement, and adjust programs for changes in the populations that they serve [4].

These factors are especially important for hospitals and the acute care that they provide to local communities. The use of hospital inpatient beds is closely related to local populations. These populations and health care providers contribute to hospital admission and discharge rates [5].

In the United States, an important influence on the use of hospital inpatient services has been the respiratory disease season. Historically, increases in the occurrence of influenza, pneumonia, and other respiratory medicine diseases have caused hospital admissions and inpatient censuses at the community level to increase [6] [7].

For health care managers and clinicians, the respiratory disease season has required increased use of resources to address the variability of inpatient admissions and censuses. Effective management of this adult medicine utilization can contribute to the efficiency and effectiveness of hospitals and other providers of care.

\section{Population}

This study focused on the respiratory disease season in the hospitals of Syracuse, New York. This area includes three large urban acute care facilities, Crouse Hospital (19,611 inpatient discharges excluding well newborns, 2018), St. Joseph's Hospital Health Center (25,394 inpatient discharges, 2018), and Upstate University hospital (32,877 inpatient discharges, 2018).

These hospitals provide primary and secondary acute care to an immediate service area of approximately 600,000 . They also provide tertiary services to the eleven county Central New York Health Service Area.

Historically, the Syracuse hospitals have maintained a conservative inpatient admission rate that has contributed to the efficiency of care in the service area. This admission rate has supported the need for movement of inpatients through these facilities in a timely manner.

The Syracuse hospitals have worked cooperatively to support efficiency with respect to inpatient utilization through monitoring inpatient admissions and reduction of inpatient lengths of stays. This has resulted in the development of community wide programs through the Hospital Executive Council [8].

\section{Method}

This study evaluated the onset and duration of the respiratory disease season in the hospitals of Syracuse, New York. It focused on inpatient utilization for these 
conditions in the combined hospitals during a five year period. Data for the study were collected and analyzed by the Hospital Executive Council.

For this study, respiratory diseases were defined as hospital adult medicine inpatients assigned to Major Diagnostic Category Four of the All Patients Refined Diagnosis Related Groups for adult medicine conditions. These patients had a principal diagnosis of a respiratory disease according to the International Classification of Diseases Ninth or Tenth Revision. The principal diagnoses were used to define this population because they were the most important influences concerning the admission of patients and the implementation of inpatient care. Patients who did not have a respiratory disease as a principal diagnosis were excluded from this study.

In the Syracuse hospitals, increased levels of respiratory diseases are generated by influenza, pneumonia, and related diagnoses. These diagnoses are related to increased levels of utilization in the hospitals between October and March of each year.

The socioeconomic characteristics of the patients included in the study were identified by the primary hospital payors of their admissions. During the five year period, Medicare accounted for 66 - 69 percent of annual admissions; Medicaid accounted for 14 - 16 percent of annual admissions; and commercial insurance accounted for 13 - 15 percent of annual admissions.

Additional analysis indicated that the numbers of inpatient hospital readmissions within 30 days for the study populations ranged from 675 to 746 between the first and last twelve-month of the study. They accounted for readmissions rates between 17.7 and 19.0 percent.

The study data were identified by month for the twelve-month periods July 2014-June 2015 through June 2018-July 2019. The twelve-month periods were employed in order to provide continuity at the beginning and end of each calendar year rather than dividing these intervals between December and January.

The initial component of the study included the respiratory disease average daily census of the combined Syracuse hospitals by month for each of the five twelve-month periods. The average daily census was defined as the total number of inpatient days for patients discharged each month divided by the total number of days in the month. By evaluating average daily census levels, the study provided an approximation of differences in health care expenses for each month.

Within each twelve-month period, the months with the six highest average daily censuses were ranked. Within each year, this period was assumed to be the respiratory disease season. The differences between the average daily census and the average daily census for the twelve-month period were also identified.

The second component of the study focused on adult medicine respiratory disease admissions/discharges for the same twelve months within each of the five years. This analysis generated information concerning numbers of patients admitted for inpatient care. It included the same procedures for identification of the six months with the highest utilization that were applied to the census data. Evaluation of this indicator provided an estimate of the resources provided to 
the hospitals for care of these patients.

Evaluation of monthly respiratory disease utilization in the first component of the study and admissions/discharges in the second component helped identify similarities and differences among the respiratory disease seasons during the five years of the study. Additional analyses of these data, such as the potential impact of changes in hospital lengths of stay, were also carried out.

\section{Results}

The first component of the study focused on average daily census levels by month for adult medicine patients with respiratory diseases in the Syracuse hospitals by month during the five-year period. Relevant data are summarized in Table 1.

This information indicated that the highest adult medicine average daily census levels for respiratory diseases in the hospitals occurred during the first quarter of each calendar year. In four of the five years, three of the six highest census levels occurred between January and March. During these three months, respiratory disease census levels in the combined hospital were at least ten patients higher than the annual means in four of the five periods.

The study data clearly demonstrated that the highest levels of respiratory disease inpatients occurred during January. This month accounted for the highest census

Table 1. Hospital inpatient average daily census, respiratory medicine (APR DRGs 130-145), Syracuse Hospitals, July 2014-June 2019.

\begin{tabular}{|c|c|c|c|c|c|c|c|c|c|c|c|c|c|}
\hline & Jul. & Aug. & Sept. & Oct. & Nov. & Dec. & Jan. & Feb. & Mar. & Apr. & May & Jun. & $\begin{array}{c}\text { Average Daily } \\
\text { Census by Month }\end{array}$ \\
\hline $2014-2015$ & 76.3 & 64.2 & 83.7 & 81.3 & 69.1 & 90.1 & 98.1 & 92.8 & 93.6 & 89.2 & 80.4 & 73.0 & 82.7 \\
\hline $2015-2016$ & 60.9 & 54.0 & 68.2 & 80.8 & 63.5 & 73.9 & 75.8 & 79.6 & 85.9 & 79.1 & 66.1 & 60.9 & 70.7 \\
\hline $2016-2017$ & 65.7 & 59.3 & 64.7 & 72.5 & 68.3 & 66.3 & 93.5 & 83.3 & 82.8 & 82.3 & 75.5 & 72.8 & 73.9 \\
\hline $2017-2018$ & 60.5 & 63.5 & 70.8 & 65.2 & 80.7 & 78.5 & 100.1 & 98.6 & 74.5 & 86.8 & 89.6 & 76.7 & 78.8 \\
\hline \multirow[t]{3}{*}{ 2018-2019 } & 74.5 & 66.5 & 64.5 & 79.3 & 79.7 & 80.4 & 92.0 & 93.5 & 93.5 & 84.8 & 78.7 & 72.1 & 80.0 \\
\hline & \multicolumn{13}{|c|}{ Ranking of Highest Volume Average Daily Censuses by Month } \\
\hline & Jul. & Aug. & Sept. & Oct. & Nov. & Dec. & Jan. & Feb. & Mar. & Apr. & May & Jun. & \\
\hline \multirow[t]{2}{*}{ 2014-2015 } & & & 6 & & & 4 & 1 & 3 & 2 & 5 & & & \\
\hline & & & $(1.3)$ & & & $(8.3)$ & $(15.8)$ & $(11.0)$ & $(11.8)$ & $(7.4)$ & & & \\
\hline \multirow[t]{2}{*}{ 2015-2016 } & & & & 2 & & 6 & 5 & 3 & 1 & 4 & & & \\
\hline & & & & $(12.5)$ & & $(4.3)$ & $(6.8)$ & $(11.1)$ & $(17.7)$ & (10.6) & & & \\
\hline \multirow[t]{2}{*}{ 2016-2017 } & & & & & & & 1 & 2 & 3 & 4 & 5 & 6 & \\
\hline & & & & & & & $(21.0)$ & $(11.2)$ & $(10.7)$ & $(10.2)$ & $(2.1)$ & $(-0.0)$ & \\
\hline \multirow[t]{2}{*}{$2017-2018$} & & & & & 5 & 6 & 1 & 2 & & 4 & 3 & & \\
\hline & & & & & $(2.3)$ & $(-0.0)$ & $(21.3)$ & $(20.0)$ & & $(9.2)$ & (12.1) & & \\
\hline \multirow[t]{2}{*}{ 2018-2019 } & & & & & 6 & 5 & 3 & 2 & 1 & 4 & & & \\
\hline & & & & & $(-0.0)$ & $(0.5)$ & $(13.1)$ & $(14.4)$ & $(14.5)$ & (5.7) & & & \\
\hline
\end{tabular}

Data include patients aged 18 and over. Data in parenthesis represent the percent increase above the annual means. Source: Hospital Executive Council. 
levels in three of the six-time periods, more than 15 patients above the annual means. In two of the periods, the January censuses were more than 20 patients higher than the annual mean levels.

Respiratory diseases census levels in the second quarter of each year, April-June, were considerably lower, accounting for three months of one year, two months of one year, and one month of the remaining three years. These amounted to reductions from utilization in the first quarter.

Census levels in the fourth-quarter, October-December, accounted for two months in three years and one month of the remaining two years. These were followed by considerable increases during January in most of the twelve-month periods.

It should be noted that the utilization data for average daily census levels in the Syracuse hospitals were influenced by differences in hospital lengths of stay. As a result of efforts to improve the efficiency of inpatient care, mean lengths of stay in the twelve-month populations of the study declined from 5.8 to 5.0 days and stays for months with the six highest average daily census levels declined from 5.8 to 5.1 days between 2014-2015 and 2018-2019. These reductions had some impact on average daily census levels by 2018-2019.

The second component of the study focused on adult medicine respiratory disease admissions and discharges during the five twelve-month periods in the combined Syracuse hospitals. Relevant data are summarized in Table 2.

Table 2. Hospital inpatient discharges, respiratory medicine (APR DRGs 130-145), Syracuse Hospitals, July 2014-June 2019.

\begin{tabular}{|c|c|c|c|c|c|c|c|c|c|c|c|c|c|}
\hline & Jul & Aug & Sept & Oct & Nov & Dec & Jan & Feb & Mar & Apr & May & Jun & Total \\
\hline 2014-2015 & 399 & 352 & 393 & 428 & 346 & 474 & 515 & 451 & 469 & 487 & 455 & 409 & 5178 \\
\hline $2015-2016$ & 368 & 303 & 356 & 455 & 396 & 435 & 429 & 434 & 537 & 463 & 406 & 372 & 4954 \\
\hline $2016-2017$ & 387 & 322 & 341 & 397 & 383 & 413 & 528 & 473 & 480 & 444 & 452 & 474 & 5094 \\
\hline $2017-2018$ & 391 & 401 & 445 & 447 & 442 & 455 & 599 & 501 & 496 & 528 & 513 & 448 & 5666 \\
\hline \multirow[t]{3}{*}{ 2018-2019 } & 459 & 416 & 391 & 483 & 453 & 500 & 559 & 504 & 550 & 522 & 493 & 457 & 5787 \\
\hline & \multicolumn{13}{|c|}{ Ranking of Highest Volume Discharges by Month Adjusted to 31 Days/Month } \\
\hline & Jul & Aug & Sept & Oct & Nov & Dec & Jan & Feb & Mar & Apr & May & Jun & \\
\hline \multirow[t]{2}{*}{ 2014-2015 } & & & & & & 4 & 1 & 3 & 5 & 2 & 6 & & \\
\hline & & & & & & $(7.7)$ & $(17.1)$ & $(13.4)$ & $(6.6)$ & $(14.3)$ & $(3.4)$ & & \\
\hline \multirow[t]{2}{*}{$2015-2016$} & & & & 4 & & 5 & 6 & 2 & 1 & 3 & & & \\
\hline & & & & $(8.1)$ & & (3.3) & (1.9) & (14.0) & $(27.6)$ & $(13.5)$ & & & \\
\hline \multirow[t]{2}{*}{ 2016-2017 } & & & & & & & 1 & 2 & 4 & 5 & 6 & 3 & \\
\hline & & & & & & & (21.9) & (21.0) & $(10.9)$ & $(6.0)$ & $(4.4)$ & (13.2) & \\
\hline \multirow[t]{2}{*}{$2017-2018$} & & & & & & & 1 & 2 & 5 & 3 & 4 & 6 & \\
\hline & & & & & & & $(24.3)$ & (15.2) & (2.9) & $(13.1)$ & $(6.4)$ & $(-0.0)$ & \\
\hline \multirow[t]{2}{*}{ 2018-2019 } & & & & & & 5 & 1 & 2 & 3 & 4 & 6 & & \\
\hline & & & & & & (1.6) & (13.6) & (13.4) & (11.8) & $(9.6)$ & $(0.2)$ & & \\
\hline
\end{tabular}

Data include patients aged 18 and over. Data in parenthesis represent the percent increase above the annual means. Source: Hospital Executive Council. 
This information demonstrated that the highest admission/discharge levels occurred between January and March of all of the time periods. In three of the time periods, respiratory disease discharges were at least six percent higher than the annual monthly averages for the hospitals. In two months, they were at least 10 percent higher. The differences between these levels and the twelve month averages were notable, but lower than those of the average daily census levels.

The study data also demonstrated that the highest levels of admission/discharge rates occurred during January. In four of the five twelve month periods, January produced the highest utilization among the five time periods for this utilization. In two of the time periods, admission/discharge levels for January were more than twenty percent higher than the twelve month averages.

Respiratory disease admission/discharge rates between April and June were lower than those for January-March. In four of the twelve month periods, the rates for April-June were still among the six highest identified. This utilization included five months with rates among the three highest.

In the fourth quarter, admission/discharge rates for respiratory diseases were considerably lower. Only four months with rates among the highest were identified. None of these was ranked among the three highest.

\section{Discussion}

Historically, the respiratory disease season, including influenza, pneumonia, and other diagnoses, has challenged populations in the United States. It has also challenged health care providers in their efforts to care for these populations. The variability of this phenomenon at the community level has required hospitals and other providers to develop and manage clinical resources.

This study reviewed the utilization of inpatient hospitals in the metropolitan area of Syracuse, New York with respect to respiratory diseases during the most recent twelve-month periods for which monthly data were available. It focused on identifying the onset and duration of the respiratory disease season by month.

The study demonstrated that, between July-June 2014-2015 and 2018-2019, inpatient respiratory diseases in the Syracuse hospitals were concentrated in the period between January and March. Within this interval, average daily inpatient census levels and numbers of admissions/discharges were the highest. The study also demonstrated that the highest levels of respiratory disease consistently occurred during January.

The study also demonstrated that the onset of the respiratory disease season was comparatively rapid, as demonstrated by the increases in census levels and discharges between October-December and January-March. The data showed that once established, increased inpatient levels of respiratory diseases extended between January-March and into April and May based on the hospital census levels as an approximation of the use of resources, such as nursing staff and medications. 
The study suggested that the highest demands for inpatient care in Syracuse increased by a community wide average daily census of $15-20$ patients during January and remained about 10 patients higher into March and April. This information could be used by health care planners and managers to address the increased needs for care during these periods.

This approach to the utilization of health care provided a framework for addressing the onset and the duration of the respiratory disease season in the Syracuse hospitals. It could be employed by other providers to identify and address these issues in their communities.

\section{Conflicts of Interest}

The authors declare no conflicts of interest regarding the publication of this paper.

\section{References}

[1] Hyman, H.H. (1982) Health Planning: A Systematic Approach. Oxford Publishing, London.

[2] Tedeschi, P.I., Wolfe, R.A. and Griffith, J.R. (1990) Micro Area Variation in Hospital Use. Inquiry, 24, 729-740.

[3] Chassin, M.R., Brook, R.H., Park, R.E., Kessey, J., Fink, A., Kahn, K., Merrick, N. and Solomon, D.H. (1986) Variations in the Use of Medical and Surgical Services by the Medicaid Population. New England Journal of Medicine, 315, 285-290. https://doi.org/10.1056/NEJM198601303140505

[4] Knickman, J.R. and Foltz, A.M. (1985) A Statistical Analysis of Reasons for East West Differences in Hospital Use. Inquiry, 22, 45-58.

[5] Shah, B.R., Reed, S.D., Francis, J., Ridley, D.B. and Schulman, K.A. (2003) The Cost of Inefficiency in U.S. Hospitals. Journal of Health Care Finance, 30, 1-9.

[6] Johnson, S.R. (2018) Hospitals Facing Financial Hit as Flu Season Continues to Worsen. Modern Healthcare, February 3, 2018.

[7] McNeil Jr., D.G. (2018) Flu Patients Arrive in Droves, and a Hospital Rolls Out the "Serge Tent". The New York Times, February 2, 2018.

[8] Lagoe, R., Pasinski, T., Kronenberg, P., Quinn, T. and Schaengold, P. (2006) Linking Health Services at the Community Level. Canada Health Care Quarterly, 9, 60-65. https://doi.org/10.12927/hcq..18229 